\title{
Pressure Analysis for Volume Fracturing Vertical Well considering Low-Velocity Non-Darcy Flow and Stress Sensitivity
}

\author{
Zhongwei Wu $\mathbb{D},{ }^{1,2}$ Chuanzhi Cui $\mathbb{D}^{1},{ }^{1}$ Japan Trivedi, ${ }^{2}$ Ning Ai, $^{3}$ and Wenhao Tang ${ }^{4}$ \\ ${ }^{1}$ College of Petroleum Engineering, China University of Petroleum (East China), No. 66 Changjiang West Road, Qingdao 266580, \\ Shangdong, China \\ ${ }^{2}$ School of Mining and Petroleum, Department of Civil and Environmental Engineering, University of Alberta, Edmonton, AB, \\ Canada T6G 2R3 \\ ${ }^{3}$ Geological Survey of Ningxia, Yinchuan, 750021 Ningxia, China \\ ${ }^{4}$ Hebei Scoilmic Petroleum Technology Co., Ltd., Chanzhou, 061001 Hebei, China
}

Correspondence should be addressed to Chuanzhi Cui; ccz2008@126.com

Received 2 April 2019; Revised 23 May 2019; Accepted 21 October 2019; Published 19 November 2019

Academic Editor: Nicoló Colombani

Copyright (C) 2019 Zhongwei Wu et al. This is an open access article distributed under the Creative Commons Attribution License, which permits unrestricted use, distribution, and reproduction in any medium, provided the original work is properly cited.

\begin{abstract}
In general, there is stress sensitivity damage in tight reservoirs and fractures. Furthermore, the flow in tight reservoirs is the lowvelocity non-Darcy flow. Currently, few researches of pressure analysis for volume fracturing vertical well are conducted simultaneously considering the low-velocity non-Darcy flow and stress sensitivity. In the paper, a novel flow model of a volume fractured vertical well is proposed and solved numerically. Firstly, the threshold pressure gradient, permeability modulus, and experimental data are, respectively, utilized to characterize the low-velocity non-Darcy flow, matrix stress sensitivity, and fracture stress sensitivity. Then, a two-region composite reservoir is established to simulate the vertical well with volume fracturing. After that, the logarithm meshing method is used to discrete the composite reservoir, and the flow model is solved by the method of finite difference and IMPES. Finally, the model verification is conducted, and the effects of the low-velocity nonDarcy flow and stress sensitivity on the pressure and pressure derivative are analyzed. The six flow regimes are identified by the dimensionless pressure and pressure derivative curve. They are, respectively, the fracture linear flow regime, early transition flow regime, radial flow regime, crossflow regime, advanced transition flow regime, and boundary controlling flow regime. The stress sensitivity and threshold pressure gradient have a great effect on the dimensionless pressure and pressure derivative. With the increase of reservoir stress sensitivity, the pressure and pressure derivative are upward at the advanced transition flow and boundary controlling regimes. However, the pressure and pressure derivative are downward at the advanced transition flow and boundary controlling regimes when the fracture sensitivity increases. An increase in the threshold pressure gradient results in a high dimensionless pressure and pressure derivative. This work reveals the effects of low-velocity non-Darcy flow and stress sensitivity on pressure and provides a more accurate reference for reservoir engineers in pressure analysis when developing a tight reservoir by using the volume fracturing vertical well.
\end{abstract}

\section{Introduction}

Volume fracturing breaks up effective permeable reservoirs to form a fracture network, maximizes the contact area between fracture sides and reservoir matrix, minimizes the fluid seepage distance of oil and gas from the matrix in any directions to fractures, and greatly improves the entire reservoir permeability [1]. Hydraulic fracture technology is an effective method to exploit unconventional reservoirs and is reported in many works [2-5], especially for volume fracturing [6-8]. Many microfractures are developed in the tight reservoir after volume fracturing $[9,10]$. During depletion, reservoir pressure changes could lead to stress variations which further alter the fracture apertures, pore, and throats, finally changing microfracture permeability and reservoir permeability [11]. In other words, the fracture and reservoir 
all are stress sensitivity medium [12-14]. In addition, due to the tiny porous and ultralow permeability of tight reservoirs, the flow in tight reservoirs obeys the law of low-velocity nonDarcy flow instead of Darcy flow [15], which is the fact that the flow velocity in a low pressure gradient regime is lower than what is estimated from Darcy's law [16].

Currently, there are many researches of the volume fracturing. Wang et al. [17] and Lu et al. [18] proposed a semianalytical model to analyze the productivity and pressure of a volume fractured vertical well in tight reservoirs. In their research, the reservoir consists of two regions, one of which is the main fracture. Another is a circular stimulated reservoir volume (SRV), which is idealized to a Warren-Root model [19]. The unstimulated reservoir volume (un-SRV) is not contributed to the flow. That is different from the actual situation, and the stress sensitivity and low-velocity nonDarcy flow are ignored. Zhu et al. [20] gave an analytical pressure solution for the volume fractured vertical well with a rectangular SRV. In their research, the reservoir is composed of multiple linear flow regions, which include SRV and un-SRV. Unfortunately, they do not consider the effect of stress sensitivity and low-velocity non-Darcy flow. Su et al. [21] proposed a fractal analytical model of volume fractured vertical wells. In their research, the whole reservoir is divided into two regions, one of which is an SRV and another is an un-SRV. The flow obeys the Darcy, and the permeability is not a function of effective pressure, which deviates the actual situation in the tight reservoir. Zhang et al. [22] proposed a semianalytical model for vertically fractured wells with stimulated reservoir volumes (SRV). The fractal porosity and permeability are employed to describe the heterogeneous distribution of porous media in the SRV. But the stress sensitivity and low-velocity nonDarcy flow are ignored. The researches mentioned above are all about volume fracturing, but they do not take the effect of the stress sensitivity and low-velocity non-Darcy flow into consideration.

Guo et al. [23] proposed a transient mathematical model for horizontal wells with consideration of the threshold pressure and nonlinear flow. In their research, the nonlinear flow is high-velocity nonlinear flow, which is different from that caused by low-velocity mentioned in this paper. And their work is just applied to the multistage fractured horizontal well. Wang et al. [24] conducted research of the transient pressure of the volume fractured vertical well. In his research, the two-region composite model is built. The inner region represents a circle SRV and is idealized as a dual-porosity medium, while the outer zone represents an un-SRV and is idealized as a single porosity reservoir. However, the lowvelocity non-Darcy flow and the stress sensitivity of fracture medium are ignored. Ji et al. [25] present a new analytical model for MFHW in tight oil reservoirs where the reservoir is subdivided into five continuous linear flow regions. The effects of non-Darcy flow and stress sensitivity are both considered. However, his research is just applied to the multistage fractured horizontal well. By considering the effect of the stress sensitivity and low-velocity non-Darcy flow, Wu et al. [26] proposed a pressure analysis model for a multistage fractured horizontal well in the tight reservoir. In his research, the tight reservoir is divided into 4 regions, which are region I to region IV. The medium of each region is stress sensitivity, and the flow in the unstimulated reservoir region obeys the low-velocity non-Darcy law. But his research is just for the multistage fractured horizontal well instead of the volume fracturing vertical well. Wu et al. [27] conduct another research of transient pressure. In this work, the fracture is discrete into many subsections. The superposition principle is utilized to build the semianalytical pressure analysis model of a multistage fracturing horizontal well considering the stress sensitivity of fracture medium. However, the reservoir is an ordinary medium instead of stress sensitivity medium in their research, and the low-velocity non-Darcy flow is ignored.

As stated above, few works simultaneously considering the effect of the low-velocity non-Darcy flow and stress sensitivity are conducted for the volume fracturing well. This paper fills this gap. To this end, the researches of the flow model of the volume fracturing well and its verification are conducted in Section 2. After that, the result and discussion are presented in Section 3. In Section 4, conclusions are presented.

\section{The Flow Model and Verification}

2.1. Physical Model of Volume Fracturing Vertical Well. According to microseismic monitoring mapping [24] and the result of indoor core fracturing experiment $[28,29]$, we can know that the reservoir after volume fracturing can be divided into two regions, one is the SRV and another is the un-SRV. The SRV is circular, and many fractures are developed in the SRV (Figure 1).

As the statement above, the physical model of the vertical well with volume fracturing can be obtained (Figure 2). As seen from Figure 2, the tight reservoir is composed of two regions, which are, respectively, the SRV and un-SRV. The un-SRV is treated as a single porosity medium. And the SRV is idealized by double porosity medium [19]. The un-SRV is stress sensitivity medium, and the flow in unSRV obeys the low-velocity non-Darcy flow. In SRV, the microfracture is developed, and all microfractures are crossed through the vertical well and penetrated the whole reservoir. The fracture permeability is a function of pressure. The relationship of fracture permeability and pressure is obtained from the experimental data of Lei et al.'s work [30]. The flow in the microfracture obeys the Darcy flow law, while the matrix is stress sensitivity medium, and the fluid steadily flows from the matrix to the microfracture and then flows to the well. The top and bottom of the reservoir are the closure. The SRV matrix and un-SRV matrix have the same reservoir properties, which include permeability, porosity, and medium stress sensitivity. The pressure and flow rate in the inner boundary of un-SRV, respectively, equal to that in the outer boundary of SRV. The flow rate of the volume fractured well is constant. The reservoir outer boundary is the closure. The reservoir and fracture are tiny compressible medium, and the process of fluid flow is isothermal. 


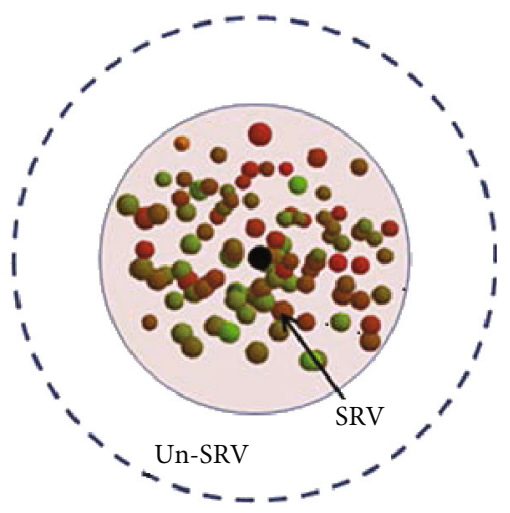

(a) Microseismic monitoring mapping [24]

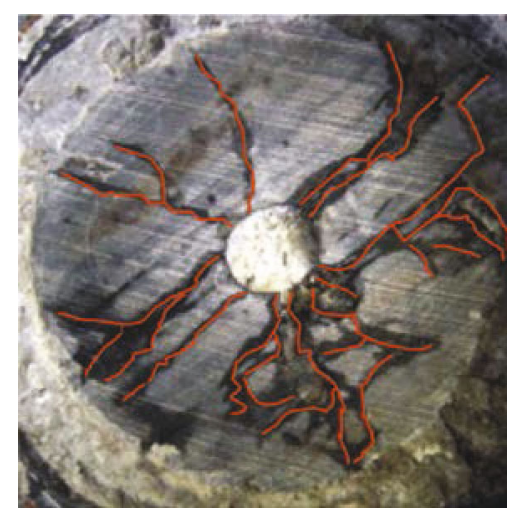

(b) Fracture morphology of unconventional reservoir core [28, 29]

FIGURE 1: The reservoir morphology after vertically fractured.

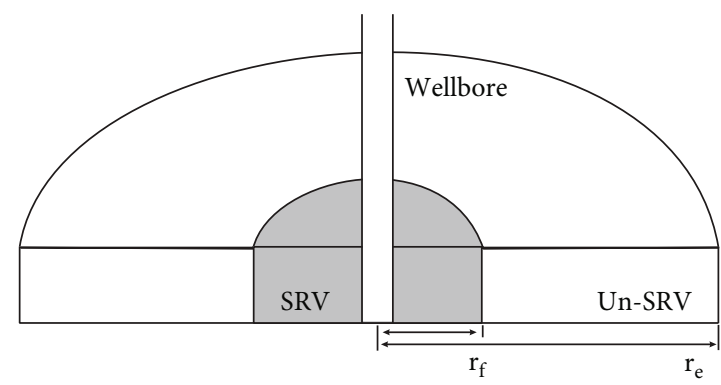

(a) 3D schematic

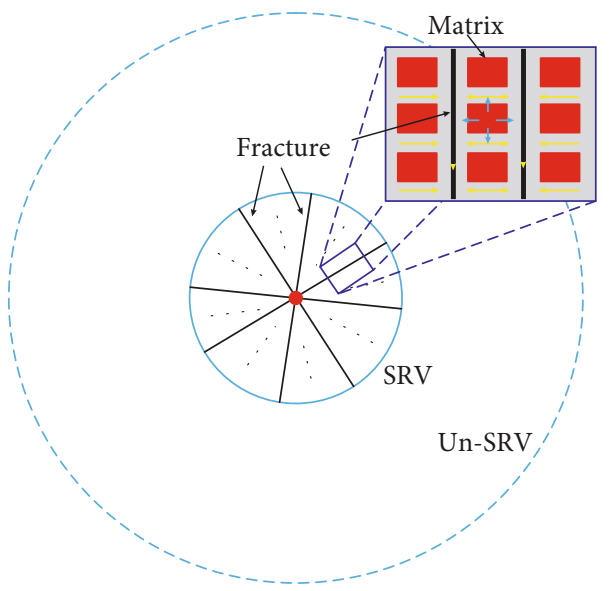

(b) Horizontal cross-section view

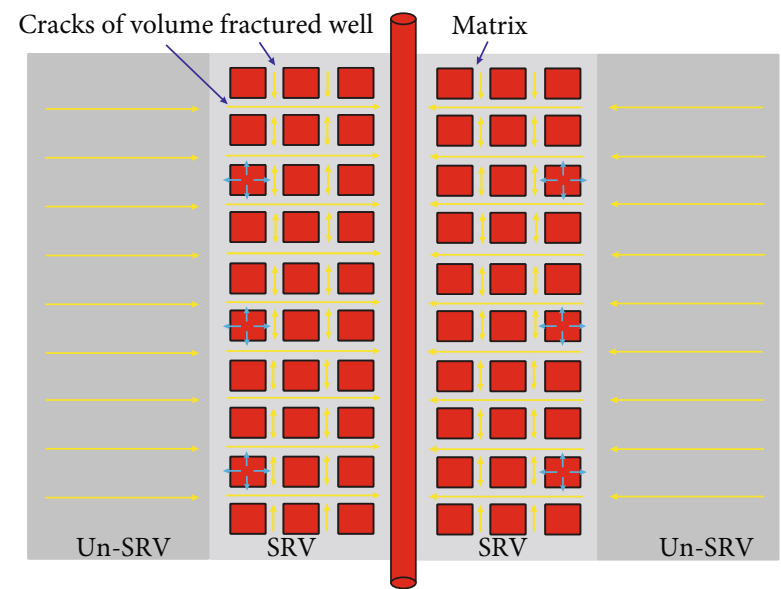

(c) Vertical cross-section view

Figure 2: Schematic for the physical model of the volume fracturing vertical well.

2.2. Mathematical Model of Volume Fracturing Vertical Well. For un-SRV, the mass conservation equation is as follows:

$$
\frac{1}{r} \frac{\partial}{\partial r}\left(-r \rho v_{\mathrm{M}}\right)=\frac{\partial}{\partial t}\left(\phi_{\mathrm{M}} \rho\right)
$$

where $\rho$ is the fluid density $\left(\mathrm{g} / \mathrm{cm}^{3}\right), r$ is the radial distance $(\mathrm{cm}), v_{\mathrm{M}}$ is the flow velocity in the un-SRV region $(\mathrm{cm} / \mathrm{s})$, $\phi_{M}$ is the porosity of the un-SRV region (dimensionless), and $t$ is the simulation time (s).
Considering low-velocity non-Darcy flow, the flow equation is as follows:

$$
v_{\mathrm{M}}=-\frac{k_{\mathrm{M}}}{\mu}\left(\frac{\partial p_{\mathrm{M}}}{\partial r}-G\right)
$$

where $\mu$ is the fluid viscosity (mPa.s), $k_{\mathrm{M}}$ is the permeability of the un-SRV region $(\mathrm{mD}), p_{\mathrm{M}}$ is the pressure in the unSRV region $\left(10^{5} \mathrm{~Pa}\right)$, and $G$ is the threshold pressure gradient $\left(10^{5} \mathrm{~Pa} / \mathrm{cm}\right)$. 
Substituting Equation (2) into Equation (1), we can obtain the flow controlling equation in un-SRV as follows:

$$
\frac{1}{r} \frac{\partial}{\partial r}\left(r \rho \frac{k_{\mathrm{M}}}{\mu}\left(\frac{\partial p_{\mathrm{M}}}{\partial r}-G\right)\right)=\frac{\partial}{\partial t}\left(\phi_{\mathrm{M}} \rho\right) .
$$

According to the microcompressible characteristic of unSRV and fluid, we can obtain the expressions of the compression factor as follows:

$$
\begin{gathered}
c_{\mathrm{M}}=\frac{1}{\phi_{\mathrm{M}}} \frac{\partial \phi_{\mathrm{M}}}{\partial p_{\mathrm{M}}}, \\
c_{\mathrm{l}}=\frac{1}{\rho_{\mathrm{l}}} \frac{\partial \rho_{\mathrm{l}}}{\partial p_{\mathrm{M}}},
\end{gathered}
$$

where $c_{\mathrm{M}}$ and $c_{1}$ are compressive factors of the un-SRV region and fluid $\left(10^{-5} \mathrm{~Pa} / \mathrm{cm}\right)$, respectively.

Simplifying Equation (3) by using Equations (4) and (5), the expression of the flow controlling equation is

$$
\frac{1}{r} \frac{\partial}{\partial r}\left(r \rho \frac{k_{\mathrm{M}}}{\mu}\left(\frac{\partial p_{\mathrm{M}}}{\partial r}-G\right)\right)=\phi_{\mathrm{M}} \rho\left(c_{\mathrm{M}}+c_{\mathrm{l}}\right) \frac{\partial p_{\mathrm{M}}}{\partial t} .
$$

Because the tight reservoir is stress sensitivity medium, the permeability of Equation (6) is a function of pressure, rather than a constant. The permeability of the tight reservoir can be calculated by $[24,31]$

$$
\gamma_{\mathrm{M}}=\frac{1}{k_{\mathrm{M}}} \frac{\partial k_{\mathrm{M}}}{\partial p_{\mathrm{M}}}
$$

where $\gamma_{M}$ is the permeability module of un-SRV $\left(10^{-5} \mathrm{~Pa}\right)$.

For SRV, the mass conservation equation flowing in the fracture is

$$
\frac{1}{r} \frac{\partial}{\partial r}\left(-r \rho v_{\mathrm{f}}\right)-\frac{\partial}{\partial t}\left(\phi_{\mathrm{m}} \rho\right)=\frac{\partial}{\partial t}\left(\phi_{\mathrm{f}} \rho\right)
$$

where $v_{\mathrm{f}}$ is the fluid flow velocity $(\mathrm{cm} / \mathrm{s}), \phi_{\mathrm{f}}$ is the fracture porosity (dimensionless), and $\phi_{\mathrm{m}}$ is the porosity of SRV medium (dimensionless).

The equation describing the fluid flow in the fracture is as follows:

$$
v_{\mathrm{f}}=-\frac{k_{\mathrm{f}}}{\mu} \frac{\partial p_{\mathrm{f}}}{\partial r}
$$

where $k_{\mathrm{f}}$ is the fracture permeability $(\mathrm{mD})$ and $p_{\mathrm{f}}$ is the fracture pressure $\left(10^{5} \mathrm{~Pa}\right)$.

In SRV, the controlling equation for crossflow from the matrix to fracture is

$$
\alpha \frac{k_{\mathrm{m}}}{\mu}\left(p_{\mathrm{f}}-p_{\mathrm{m}}\right)=\frac{\partial}{\partial t}\left(\phi_{\mathrm{m}} \rho\right)
$$

where $\alpha$ is the shape factor, which is utilized to characterize the flow between the matrix and the fracture (dimensionless), $k_{\mathrm{m}}$ is the permeability of SRV medium $(\mathrm{mD})$, and $p_{\mathrm{m}}$ is the medium pressure in SRV $\left(10^{5} \mathrm{~Pa}\right)$.

According to the microcompressible characteristic of the matrix and fracture in SRV, we can get two expressions as follows:

$$
\begin{gathered}
c_{\mathrm{f}}=\frac{1}{\phi_{\mathrm{f}}} \frac{\partial \phi_{\mathrm{f}}}{\partial p_{\mathrm{f}}}, \\
c_{\mathrm{m}}=\frac{1}{\phi_{\mathrm{m}}} \frac{\partial \phi_{\mathrm{m}}}{\partial p_{\mathrm{m}}},
\end{gathered}
$$

where $c_{\mathrm{f}}$ and $c_{\mathrm{m}}$ are, respectively, the compressive factors of the fracture and medium $\left(10^{-5} \mathrm{~Pa} / \mathrm{cm}\right)$.

By Equations (9)-(12), Equation (8) can be simplified to be

$$
\frac{1}{r} \frac{\partial}{\partial r}\left(r \rho \frac{k_{\mathrm{f}}}{\mu} \frac{\partial p_{\mathrm{f}}}{\partial r}\right)-\phi_{\mathrm{m}} \rho\left(c_{\mathrm{l}}+c_{\mathrm{m}}\right) \frac{\partial p_{\mathrm{m}}}{\partial t}=\phi_{\mathrm{f}} \rho\left(c_{\mathrm{l}}+c_{\mathrm{f}}\right) \frac{\partial p_{\mathrm{f}}}{\partial t}
$$

Similarly, by Equation (12), Equation (10) can be rewritten as

$$
\alpha \frac{k_{\mathrm{m}}}{\mu}\left(p_{\mathrm{f}}-p_{\mathrm{m}}\right)=\phi_{\mathrm{m}} \rho\left(c_{\mathrm{l}}+c_{\mathrm{m}}\right) \frac{\partial p_{\mathrm{m}}}{\partial t} .
$$

The permeability used in Equations (13) and (14) is not a constant, but a function of pressure. The relationship between matrix permeability in SRV and pressure is [24]

$$
\gamma_{\mathrm{m}}=\frac{1}{k_{\mathrm{m}}} \frac{\partial k_{\mathrm{m}}}{\partial p},
$$

where $\gamma_{\mathrm{m}}$ are the permeability modulus of the SRV matrix $\left(10^{-5} \mathrm{~Pa}\right)$.

From Lei et al.'s work [30], a polynomial descripting the law of stress sensitivity of fracture is as follows:

$$
\frac{k_{\mathrm{f}}}{k_{\mathrm{fi}}}=\frac{c_{0}}{100} p_{\mathrm{eff}}^{2}+\frac{c_{1}}{10} p_{\text {eff }}+c_{2},
$$

where $c_{0}, c_{1}$, and $c_{2}$ are the fitting coefficients and $p_{\text {eff }}$ is the effective stress $\left(10^{5} \mathrm{~Pa}\right)$, the expression of which is $p_{\text {eff }}=$ $p_{\text {over }}-p_{\mathrm{f}} \cdot p_{\text {over }}$ is overburdened rock stress $\left(10^{5} \mathrm{~Pa}\right)$. By fitting to the experimental data from Lei et al.'s work [30], we can obtain the values of the fitting coefficients: $c_{0}=0.25, c_{1}=$ 0.976 , and $c_{2}=1.0368$.

The connection conditions between the SRV and un-SRV are as follows:

$$
\begin{gathered}
\left.k_{\mathrm{f}} \frac{\partial p_{\mathrm{f}}}{\partial r}\right|_{r=r_{\mathrm{f}}}=\left.k_{\mathrm{M}} \frac{\partial p_{\mathrm{M}}}{\partial r}\right|_{r=r_{\mathrm{f}}}, \\
\left.p_{\mathrm{f}}\right|_{r=r_{\mathrm{f}}}=\left.p_{\mathrm{M}}\right|_{r=r_{\mathrm{f}}},
\end{gathered}
$$

where $r_{\mathrm{f}}$ is the outer boundary of SRV $(\mathrm{cm})$. 
The outer boundary condition is

$$
\left.\frac{\partial p_{\mathrm{M}}}{\partial r}\right|_{r=r_{\mathrm{e}}}=0,
$$

where $r_{\mathrm{e}}$ is the outer boundary of un-SRV $(\mathrm{cm})$.

The inner boundary condition is

$$
\left.k_{\mathrm{f}} r \frac{\partial p_{\mathrm{f}}}{\partial r}\right|_{r=r_{\mathrm{w}}}=\frac{\mu Q}{2 \pi h},
$$

where $r_{\mathrm{w}}$ is the well radius $(\mathrm{cm})$.

The initial condition is as follows:

$$
p_{\mathrm{f}}=p_{\mathrm{m}}=p_{\mathrm{M}}=p_{\mathrm{in}}
$$

where $p_{\text {in }}$ is the initial pressure $\left(10^{5} \mathrm{~Pa}\right)$.

Equations (6), (7), and (13)-(21) compose the mathematical model of the volume fractured vertical well considering the stress sensitivity and low-velocity non-Darcy flow. The next subsection introduces the model solving method.

\subsection{Model Solution and Verification}

2.3.1. Model Solution. From the type of controlling equations (Equations (6) and (13)), we can know that the controlling equations can only be simplified to be strong nonlinear and variable coefficient partial differential equations, although the Pedrosa transformation [32] is used. It is difficult to obtain the analytical solution of the partial differential equation with a strong nonlinear term and variable coefficient. So, the finite difference method [16] is used to solve the model of the volume fracturing vertical well considering the stress sensitivity and low-velocity non-Darcy flow. The flow coefficient term is treated explicitly and changed with the pressure. In order to obtain the discrete grid distribution with characteristics that the grid grows large with being far away from the well, a logarithm transformation is introduced as follows:

$$
\ln \left(\frac{r}{r_{\mathrm{w}}}\right)=x,
$$

where $x$ is a constant and is utilized to describe the meshing relationship.

By using Equation (22), the simplified Equation (6) is

$$
\begin{aligned}
& \frac{\partial}{\partial x}\left(\rho \frac{k_{\mathrm{M}}}{\mu} \frac{\partial p_{\mathrm{M}}}{\partial x}-r \rho \frac{k_{\mathrm{M}}}{\mu} G\right) \\
& =\left(r_{\mathrm{w}} e^{2 x}\right)^{2} \phi_{\mathrm{M}} \rho\left(c_{\mathrm{M}}+c_{\mathrm{l}}\right) \frac{\partial p_{\mathrm{M}}}{\partial t} .
\end{aligned}
$$

By using the finite difference algorithm and upstream weighting method, Equation (23) can be rewritten as

$$
\begin{aligned}
\frac{\lambda_{\mathrm{M}, i}^{n}}{\Delta x^{2}} p_{\mathrm{M}, i-1}^{n+1}-\frac{\lambda_{\mathrm{M}, i}^{n}+\lambda_{\mathrm{M}, i+1}^{n}}{\Delta x^{2}} p_{\mathrm{M}, i}^{n+1} & \\
& +\frac{\lambda_{\mathrm{M}, i+1}^{n}}{\Delta x^{2}} p_{\mathrm{M}, i+1}^{n+1}+r_{\mathrm{w}} e^{\Delta x \cdot i} \frac{\lambda_{\mathrm{M}, i}^{n}-\lambda_{\mathrm{M}, i+1}^{n}}{\Delta x} G \\
= & \left(r_{\mathrm{w}} e^{\Delta x \cdot i}\right)^{2}\left(\phi_{\mathrm{M}} \rho\left(c_{\mathrm{M}}+c_{l}\right)\right)_{i}^{n} \frac{p_{\mathrm{M}, i}^{n+1}-p_{\mathrm{M}, i}^{n}}{\Delta t},
\end{aligned}
$$

where $\lambda_{\mathrm{M}}=\rho\left(k_{\mathrm{M}} / \mu\right)$; superscripts $n$ and $n+1$ are the time step (s); $\Delta t$ and $\Delta x$ are, respectively, the simulation time interval (s) and discrete unit size $(\mathrm{cm})$; subscript $M$ represents the un-SRV region; and subscripts $i-1$, and $i+1$ represent the discrete grid position.

Similarly, the Equations (13) and (14) can be, respectively, rewritten as

$$
\begin{gathered}
\frac{\lambda_{\mathrm{f}, i}^{n}}{\Delta x^{2}} p_{\mathrm{f}, i-1}^{n+1}-\frac{\lambda_{\mathrm{f}, i}^{n}+\lambda_{\mathrm{f}, i+1}^{n}}{\Delta x^{2}} p_{\mathrm{f}, i}^{n+1}+\frac{\lambda_{\mathrm{f}, i+1}^{n}}{\Delta x^{2}} p_{\mathrm{f}, i+1}^{n+1} \\
-\left(r_{\mathrm{w}} e^{\Delta x \cdot i}\right)^{2}\left(\phi_{\mathrm{m}} \rho\left(c_{\mathrm{l}}+c_{\mathrm{m}}\right)\right)_{i}^{n} \frac{p_{\mathrm{m}, i}^{n+1}-p_{\mathrm{m}, i}^{n}}{\Delta t} \\
=\left(r_{\mathrm{w}} e^{\Delta x \cdot i}\right)^{2}\left(\phi_{f} \rho\left(c_{f}+c_{l}\right)\right)_{i}^{n} \frac{p_{\mathrm{f}, i}^{n+1}-p_{\mathrm{f}, i}^{n}}{\Delta t} \\
\alpha \frac{k_{\mathrm{m}}}{\mu}\left(p_{\mathrm{f}, i}^{n+1}-p_{\mathrm{m}, i}^{n+1}\right)=\left(\phi_{\mathrm{m}} \rho\left(c_{\mathrm{l}}+c_{\mathrm{m}}\right)\right)_{i}^{n} \frac{p_{\mathrm{m}, i}^{n+1}-p_{\mathrm{m}, i}^{n}}{\Delta t}
\end{gathered}
$$

where $\lambda_{\mathrm{m}}=\rho\left(k_{\mathrm{m}} / \mu\right) ; \lambda_{\mathrm{f}}=\rho\left(k_{\mathrm{f}} / \mu\right)$; and subscripts $\mathrm{f}$ and $\mathrm{m}$ are, respectively, the fracture and matrix of the SRV region.

The discrete expression of outer boundary conditions is

$$
p_{N}^{n+1}=p_{N-1}^{n+1}
$$

where $N$ is the discrete grid number of the reservoir.

The discrete expressions of the connection condition between SRV and un-SRV are

$$
\begin{aligned}
& k_{\mathrm{f}}\left(p_{\mathrm{f}, N_{1}}^{n+1}-p_{\mathrm{f}, N_{1}-1}^{n+1}\right)=k_{\mathrm{M}}\left(p_{\mathrm{M}, N_{1}+1}^{n+1}-p_{\mathrm{f}, N_{1}}^{n+1}\right), \\
&\left.p_{\mathrm{f}}\right|_{x=r_{\mathrm{w}} e^{N_{1}} \cdot \Delta x}=\left.p_{\mathrm{M}}\right|_{x=r_{\mathrm{w}} e^{N_{1} \cdot \Delta x}},
\end{aligned}
$$

where $N_{1}$ is the discrete grid number of the SRV.

The discrete expression of inner boundary conditions is

$$
k_{\mathrm{f}} \frac{p_{\mathrm{f}, 2}^{n+1}-p_{\mathrm{f}, 1}^{n+1}}{\Delta x}=\frac{\mu Q}{2 \pi h},
$$

where $\lambda_{\mathrm{m}}, \lambda_{\mathrm{f}}$, and $\lambda_{\mathrm{M}}$ are not a constant, but a function of pressure. In our simulation, we explicitly obtain the value of $\lambda_{\mathrm{m}}, \lambda_{\mathrm{f}}$, and $\lambda_{\mathrm{M}}$. In another word, the $\lambda_{\mathrm{m}}, \lambda_{\mathrm{f}}$, and $\lambda_{\mathrm{M}}$ at $(n+1)$ th time step equal to that at $n$th time step when calculated the reservoir pressure of $(n+1)$ th time. After the pressure of $(n+1)$ th time is obtained, the $\lambda_{\mathrm{m}}, \lambda_{\mathrm{f}}$, and $\lambda_{\mathrm{M}}$ at $(n+1)$ th time step are recalculated by using the pressure of $(n+1)$ th time. 
The $k_{\mathrm{m}, i}^{n}$ and $k_{\mathrm{M}, i}^{n}$ at $n$th time step are, respectively, calculated by

$$
\begin{aligned}
& k_{\mathrm{m}, i}^{n}=k_{\mathrm{m}} e^{-\gamma_{\mathrm{m}}\left(p_{\mathrm{in}}-p_{\mathrm{m}, i}^{n}\right)}, \\
& k_{\mathrm{M}, i}^{n}=k_{\mathrm{M}} e^{-\gamma_{\mathrm{M}}\left(p_{\mathrm{in}}-p_{\mathrm{M}, i}^{n}\right)},
\end{aligned}
$$

where subscript is the initial value. lows:

The permeability expression of SRV fracture $k_{\mathrm{f}}^{n}$ is as fol-

$$
k_{\mathrm{f}}^{n}=k_{\mathrm{fi}}\left(\frac{c_{0}}{100}\left(p_{\mathrm{eff}}^{n}\right)^{2}+\frac{c_{1}}{10} p_{\mathrm{eff}}^{n}+c_{2}\right) .
$$

Equations (22)-(33) compose the discrete mathematical model for the scenario of the well with a constant flow rate. The above discrete model can be solved by the Gaussian elimination method.

2.3.2. Model Verification. In order to verify our model, we assume that the circle reservoir is not fractured, namely, the reservoir physical properties of SRV are the same to that of un-SRV. And reservoir permeability is constant, and the pseudothreshold pressure gradient $(G)$ is set to zero. Finally, the proposed model can be simplified to be a conventional reservoir with the vertical well. We take a comparison between the results calculated by the simplified model and the commercial software. The simplified model is performed based on MATLAB. The grid spatial distribution of commercial software is the same as the simplified model. The schematic figure of the grid spatial distribution is shown in Figure 3. From Figure 3, we can know that the grid has nonuniform distribution. The closer to the wellbore is, the smaller the grid is.

The sizes of the simulation reservoir and SRV are, respectively, 100 and $10 \mathrm{~m}$. The discrete grid number of the simulation reservoir and SRV in the radial direction are, respectively, 20 and 13 . The flow rate of the volume fracturing vertical and other parameters used in the simulation are in Table 1. The definitions of dimensionless parameters are as follows:

$$
\begin{aligned}
t_{D} & =\frac{k_{\mathrm{in}, \mathrm{f}} t}{\phi_{\mathrm{f}}\left(c_{\mathrm{f}}+c_{\mathrm{l}}\right) \mu r_{\mathrm{w}}^{2}+\phi_{\mathrm{M}}\left(c_{\mathrm{M}}+c_{\mathrm{l}}\right) \mu r_{\mathrm{w}}^{2}}, \\
p_{D} & =\frac{2 \pi h k_{\mathrm{in}, \mathrm{f}}}{\mu \mathrm{Q}}\left(p_{\mathrm{in}}-p\right) .
\end{aligned}
$$

Using the grid distribution and parameters above, the comparing result of our model and commercial software is conducted (Figure 4). From Figure 4, we can know that the dimensionless pressure and dimensionless pressure derivative calculated by the simplified model considerately agree with that of the commercial software. That verifies the correctness of our model and its solution method. The next subsection conducts the effects of key parameters, including stress sensitivity and pseudothreshold pressure gradient, on dimensionless pressure and pressure derivative.

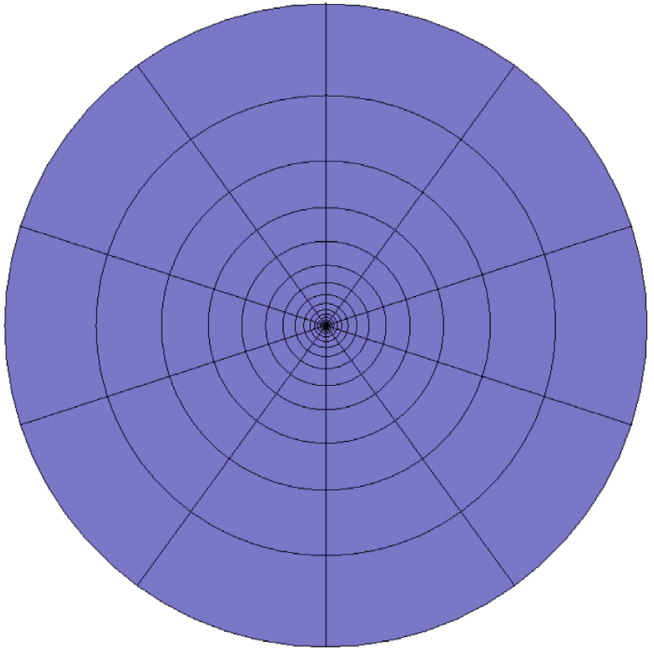

Figure 3: The schematic figure of the grid spatial distribution.

TABle 1: Parameters used in the comparison.

\begin{tabular}{lc}
\hline Parameter & Value \\
\hline Radius of reservoir under vertical well controlling $(\mathrm{cm})$ & 10000 \\
Radius of SRV $(\mathrm{cm})$ & 1000 \\
Radius of vertical well $(\mathrm{cm})$ & 12.4 \\
Reservoir thickness $(\mathrm{cm})$ & 600 \\
Reservoir permeability $(\mathrm{mD})$ & 0.23 \\
Reservoir porosity & 0.15 \\
Porosity of fracture in the SRV & 0.02 \\
Reservoir compression factor $\left(\left(10^{5} \mathrm{~Pa}\right)^{-1}\right)$ & 0.0038 \\
Compression factor of fracture in the SRV $\left(\left(10^{5} \mathrm{~Pa}\right)^{-1}\right)$ & 0.0056 \\
Fluid compression factor $\left(\left(10^{5} \mathrm{~Pa}\right)^{-1}\right)$ & 0.006 \\
Fluid viscosity $(\mathrm{mPa} \cdot \mathrm{s})$ & 5.6 \\
Fluid density $\left(\mathrm{g} / \mathrm{cm}^{3}\right)$ & 0.8 \\
Initial pressure $\left(10^{5} \mathrm{~Pa}\right)$ & 300 \\
Total grid number & 20 \\
Grid number of SRV & 13 \\
Permeability modulus of reservoir $\left(\left(10^{5} \mathrm{~Pa}\right)^{-1}\right)$ & 0 \\
Pseudothreshold pressure gradient $\left(10^{5} \mathrm{~Pa} / \mathrm{cm}\right)$ & 0 \\
Well flow rate $(\mathrm{cm} / \mathrm{s})$ & 300 \\
\hline
\end{tabular}

\section{The Results and Discussions}

3.1. Flow Regimes. In order to divide flow regimes of the volume fractured vertical well considering stress sensitivity and low-velocity non-Darcy, we set the permeability modulus of the reservoir matrix to $0.001\left(10^{5} \mathrm{~Pa}\right)^{-1}$. The pseudothreshold pressure gradient $(G)$ equals to $0.001\left(10^{5} \mathrm{~Pa} / \mathrm{cm}\right)$. The fitting factors used to descript fracture sensitivity are, respectively, $c_{0}=0.25, c_{1}=0.976$, and $c_{2}=1.0368$. Other parameters used are in Table 1. We obtain the curves of dimensionless pressure and pressure derivative, which can be seen in Figure 5.

As seen from Figure 5, there are six flow regimes presented by the type curves of the volume fractured vertical well considering stress sensitivity and low-velocity non-Darcy in the tight reservoir. Stage 1 is the fracture linear flow regime. 


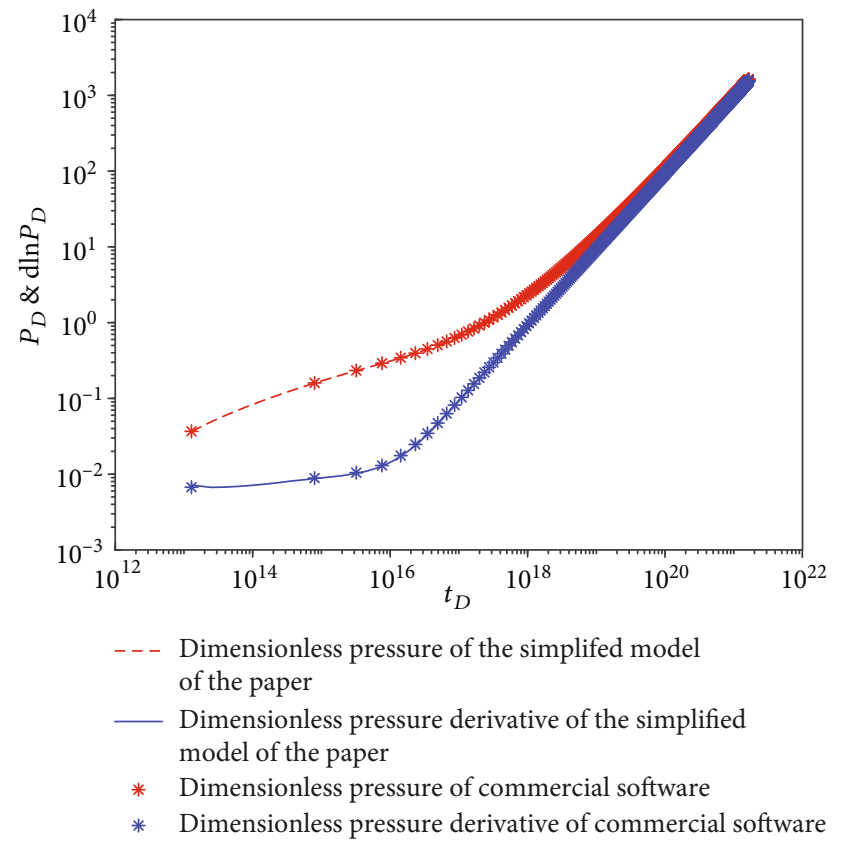

Figure 4: Comparison result between the paper model and commercial software.

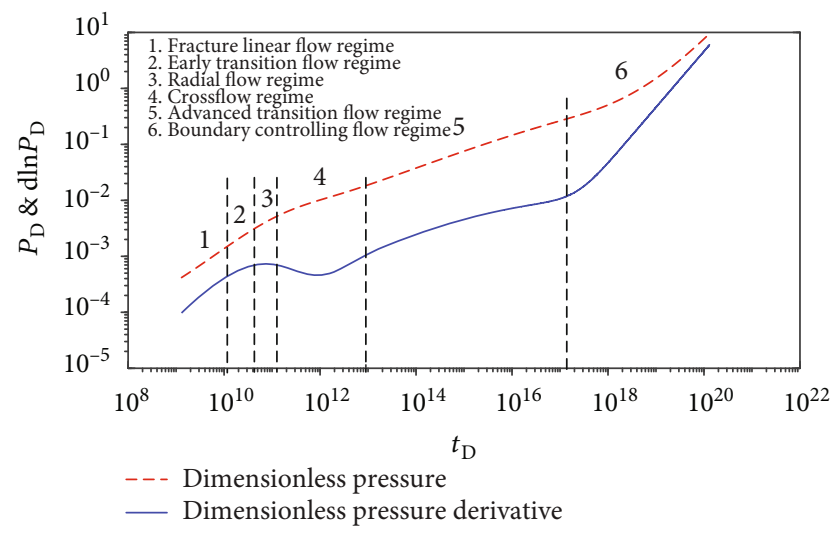

FiguRe 5: Dimensionless pressure and pressure derivative curve for dividing the flow regimes.

In this regime, the fluid flows in the fracture, and there is no interference between fractures. This flow regime is easy to be vanished. Stage 2 is the early transition flow regime [21]. The dimensionless pressure derivative goes downward in this stage. Stage 3 is the radial flow regime, in which the interferences between fractures occur, and the fluid radially flows to the wellbore. Stage 4 is the crossflow regime. With the decrease of pressure in the fracture, the pressure difference between the fracture and the matrix increases. So, the crossflow between the matrix and the fracture occurs. Stage 5 is the advanced transition flow regime. In these flow regimes, the fluid flows both in SRV and un-SRV. Because of poor reservoir physical properties of un-SRV when compared with that of SRV, the pressure derivative goes upward, which is similar to the closed boundary [21]. The stage 6 is the boundary controlling the flow regime. The dimensionless pressure

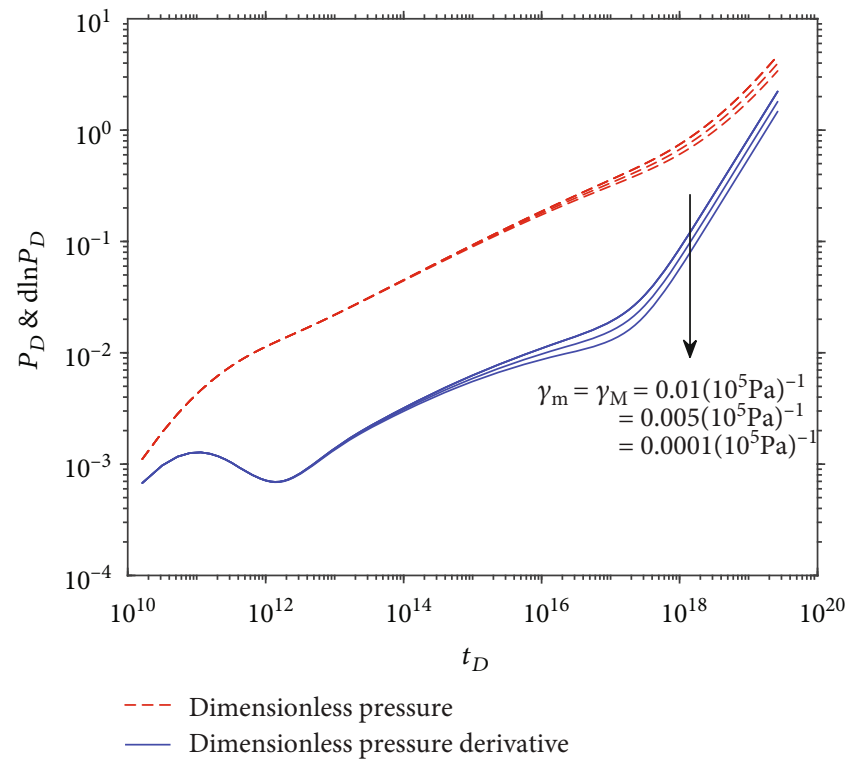

Figure 6: The effect of reservoir permeability modulus.

and pressure derivative curves go upward, and its slopes equal to 1 when the boundary is closed.

3.2. The Effects of Stress Sensitivity and Low-Velocity NonDarcy Flow. In this subsection, the effects of stress sensitivity and low-velocity non-Darcy flow on dimensionless pressure and pressure derivative are analyzed.

The fitting factors describing fracture stress sensitivity are, respectively, $c_{0}=0.25, c_{1}=0.976$, and $c_{2}=1.0368$. The pseudothreshold pressure gradient equals to 0.001 $\left(10^{5} \mathrm{~Pa} / \mathrm{cm}\right)$. The effects of reservoir permeability modulus (the value of $\gamma_{\mathrm{m}}$ and $\gamma_{\mathrm{M}}$ is the same) on dimensionless pressure and pressure derivative are analyzed and shown in Figure 6.

As seen from Figure 6, the reservoir permeability modulus mainly affects flow regimes five and six, which are, respectively, advanced transition flow regime and boundary controlling flow regime. Due to the high fracture conductivity, the pressure drop in the fracture is tiny, which results that the steady crossflowing rate between fracture and matrix is small. So, the pressure drop in the SRV matrix is small, and the matrix permeability damage is slight. However, in the advanced transition flow regime and boundary controlling flow regime, a greater pressure decreasing in the reservoir is formed. So, the reservoir permeability modulus has great effects on the dimensionless pressure and pressure derivative. The larger reservoir permeability modulus corresponds to a greater damage of the tight reservoir permeability when the same pressure drop occurs. An increase in the tight reservoir permeability damage results in an increase in the pressure drop when the flow rate is a constant. That results the dimensionless pressure and pressure derivative to go upward as shown in Figure 5.

In order to investigate the effect of fracture stress sensitivity, we obtain the fracture sensitivity data from Lei et al.'s work [30] (Figure 7). From Figure 7, the fracture stress sensitivity becomes significant from cores 1 to 3 . By fitting the 


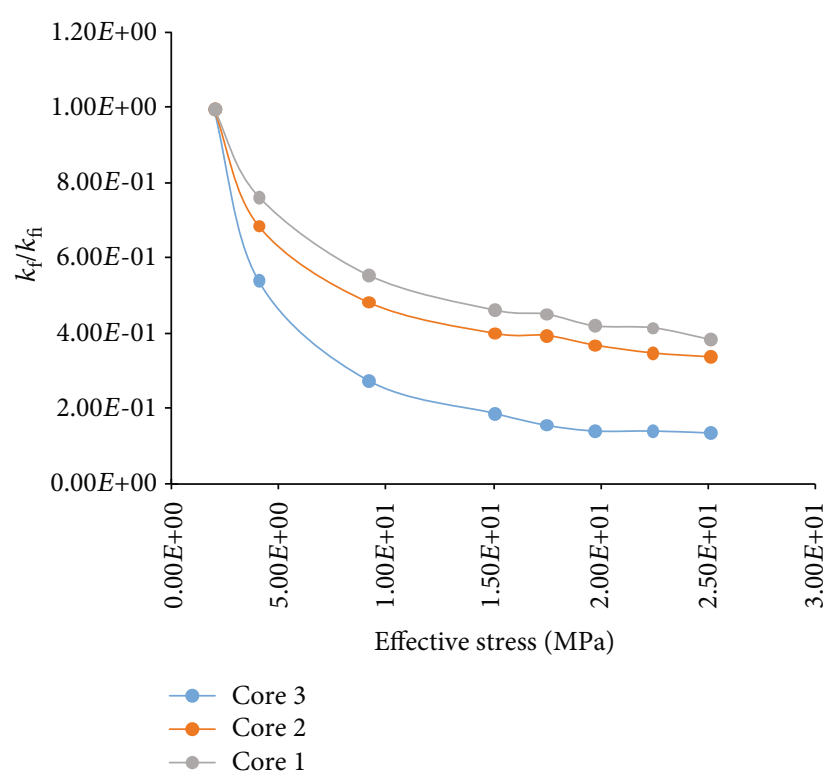

FIGURE 7: Fracture stress sensitivity data from Lei et al.'s work [30].

TABLE 2: The fitting coefficients of cores 1 to 3 .

\begin{tabular}{lccc}
\hline Core no. & $c_{0}$ & $c_{1}$ & $c_{2}$ \\
\hline 1 & 0.15 & -0.635 & 1.0625 \\
2 & 0.18 & -0.705 & 1.0456 \\
3 & 0.25 & -0.976 & 1.0368 \\
\hline
\end{tabular}

experimental data, we can obtain the fitting coefficients of cores 1 to 3 . The fitting coefficients of cores 1 to 3 can be seen in Table 2 .

By using the data in Tables 1 and 2, the effect of fracture stress sensitivity is conducted. In this sensitive analysis, the reservoir permeability modulus is $0.001\left(10^{5} \mathrm{~Pa}\right)^{-1}$. The pseudothreshold pressure gradient equals to $0.001\left(10^{5} \mathrm{~Pa} / \mathrm{cm}\right)$. The effects of fracture stress sensitivity on dimensionless pressure and pressure derivative are seen in Figure 8. From Figure 8 , we can obtain that the fracture sensitivity mainly influences the advanced transition flow regime and boundary flow regime. With the increase of fracture stress sensitivity (from cases 1 to 3 ), the dimensionless pressure and pressure derivative decrease at the advanced transition flow regime and boundary flow regime. This is due to the increase of stress sensitivity indicating an increase in the damage of fracture permeability, which results in a large pressure drop when the flow rate is constant.

The permeability modulus of the matrix is $0.001\left(10^{5} \mathrm{~Pa}\right)^{-1}$. The fitting factors used to describe fracture stress sensitivity are, respectively, $c_{0}=0.25, c_{1}=0.976$, and $c_{2}=1.0368$. And other parameters are in Table 1 . The effects of pseudothreshold pressure gradient $(G)$ on dimensionless pressure and pressure derivative are analyzed and shown in Figure 9.

From Figure 9, we get that the pseudothreshold pressure gradient has great influence on the dimensionless pressure and pressure derivative. With the increase of $G$, the dimensionless pressure and pressure derivative increase. The rea-

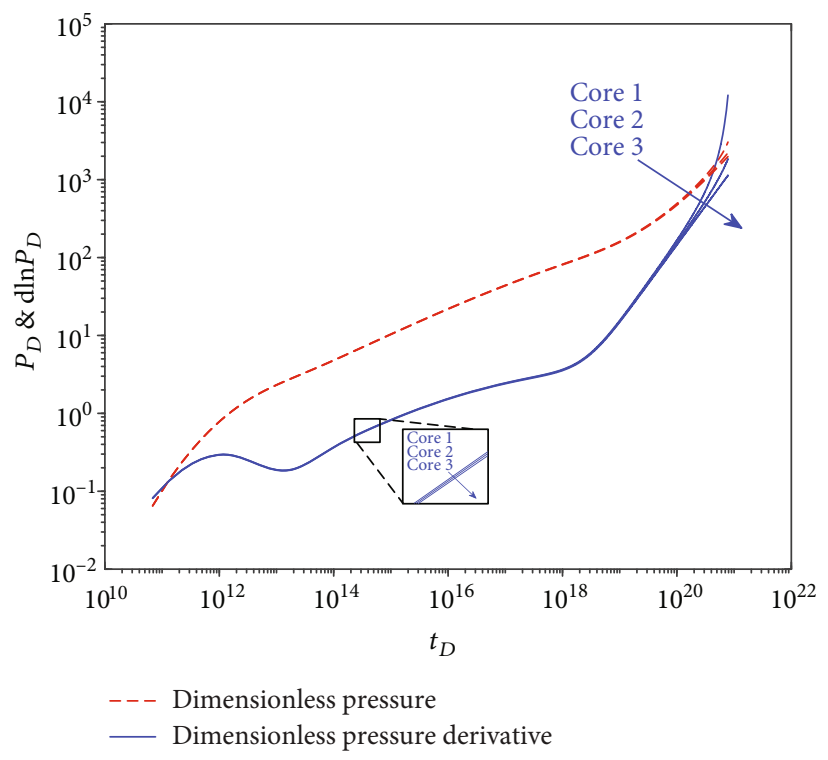

Figure 8: The effect of fracture stress sensitivity.

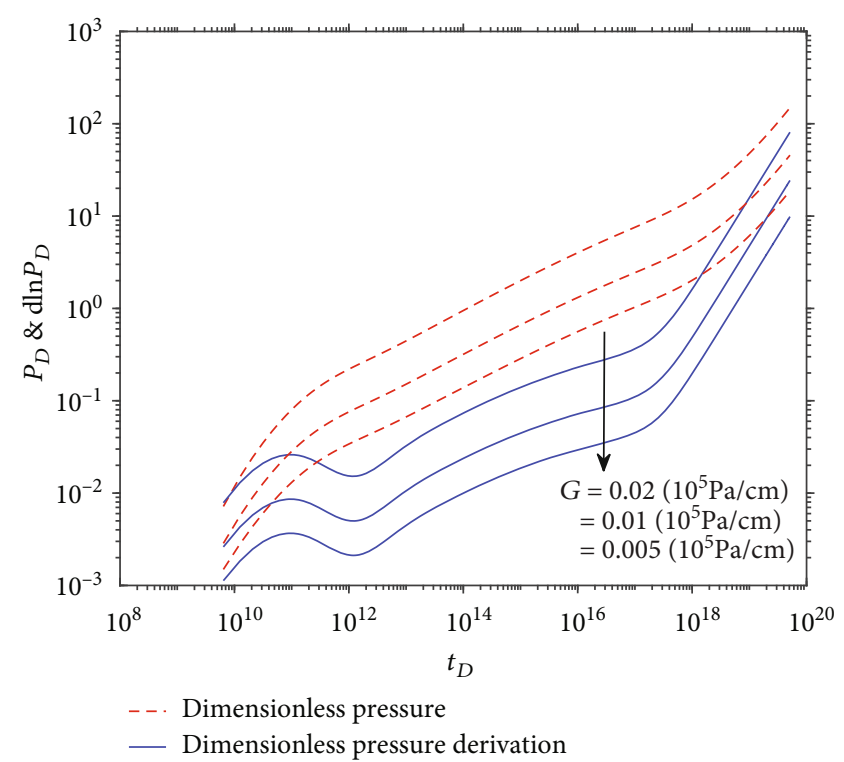

FIGURE 9: The effect of pseudothreshold pressure gradient $(G)$.

son for this case is that an increase in the pseudothreshold pressure gradient is corresponding to large flow resistance, which requires a high driving pressure to maintain a constant flow rate of the volume fracturing well. So, the dimensionless pressure and pressure derivative increase when the pseudothreshold pressure gradient increases.

\section{Conclusion}

(1) A numerical pressure analysis model of the volume fracturing vertical well considering stress sensitivity and low-velocity non-Darcy flow is proposed, and the numerical solution is obtained by a finite difference method 
(2) The flow regimes for the volume fracturing vertical well considering stress sensitivity and low-velocity non-Darcy flow can be identified by the dimensionless pressure and pressure derivative curves. Six flow stages are identified and include the fracture linear flow regime, early transition flow regime, radial flow regime, crossflow regime, advanced transition flow regime, and boundary controlling flow regime

(3) The stress sensitivity and pseudopressure gradient have a great effect on the dimensionless pressure and pressure derivative. With the increase of reservoir stress sensitivity, the pressure and pressure derivative are upward at the advanced transition flow and boundary controlling regimes. However, the pressure and pressure derivative are downward at the advanced transition flow and boundary controlling regimes when the fracture sensitivity increases. An increasing pseudopressure gradient is corresponding to high dimensionless pressure and pressure derivative

\section{Data Availability}

The data used to support the findings of this study are included within the article.

\section{Conflicts of Interest}

The authors declare that they have no conflicts of interest.

\section{Acknowledgments}

This work is supported by the Fundamental Research Funds for the Central Universities (No. 18CX06011A), National Natural Science Foundation of China (No. 51974343), China University of Petroleum (Beijing) Graduate Innovation Engineering Project (No. YCX2018013), National Massive Oil \& Gas Field and Coal-Bed Methane Development Program (No. 2016ZX05010-002-007), National Science and Technology Major Demonstration Project "Tight Oil Development Demonstration Project of Bohai Bay Basin Jiyang Depression" (No. 2017ZX05072-006-004), and Shandong Provincial Natural Science Foundation (No. ZR2017MEE054).

\section{References}

[1] Q. Wu, Y. Xu, X. Wang, T. Wang, and S. Zhang, "Volume fracturing technology of unconventional reservoirs: connotation, design optimization and implementation," Petroleum Exploration and Development, vol. 39, no. 3, pp. 377-384, 2012.

[2] J. Cai, W. Wei, X. Hu, R. Liu, and J. Wang, "Fractal characterization of dynamic fracture network extension in porous media," Fractals, vol. 25, no. 02, article 1750023, 2017.

[3] P. Tan, Y. Jin, L. Yuan et al., "Understanding hydraulic fracture propagation behavior in tight sandstone-coal interbedded formations: an experimental investigation," Petroleum Science, vol. 16, no. 1, pp. 148-160, 2019.

[4] J. Yuan, R. Jiang, and W. Zhang, "The workflow to analyze hydraulic fracture effect on hydraulic fractured horizontal well production in composite formation system," Advances in GeoEnergy Research, vol. 2, no. 3, pp. 319-342, 2018.

[5] X. Li, J. Liang, W. Xu, X. Li, and X. Tan, "The new method on gas-water two phase steady-state productivity of fractured horizontal well in tight gas reservoir," Advances in Geo-Energy Research, vol. 1, no. 2, pp. 105-111, 2017.

[6] Q. Zhang, Y. Su, H. Zhao, W. Wang, K. Zhang, and M. Lu, "Analytic evaluation method of fractal effective stimulated reservoir volume for fractured wells in unconventional gas reservoirs," Fractals, vol. 26, no. 06, article 1850097, 2018.

[7] Z. Wu, C. Cui, Y. Hao et al., "Relative permeability model taking the roughness and actual fluid distributions into consideration for water flooding reservoirs," Arabian Journal for Science and Engineering, vol. 44, no. 12, pp. 10513-10523, 2019.

[8] H. Wang, X. Liao, and H. Ding, "Monitoring and evaluating the volume fracturing effect of horizontal well," Journal of Natural Gas Science and Engineering, vol. 22, pp. 498-502, 2015.

[9] G. Sheng, Y. Su, and W. Wang, "A new fractal approach for describing induced-fracture porosity/permeability/ compressibility in stimulated unconventional reservoirs," Journal of Petroleum Science and Engineering, vol. 179, pp. 855-866, 2019.

[10] Z. Chen, X. Liao, W. Yu, and K. Sepehrnoori, "Pressure-transient behaviors of wells in fractured reservoirs with naturaland hydraulic-fracture networks," SPE Journal, vol. 24, no. 01, pp. 375-394, 2019.

[11] Y. Tian, "Experimental study on stress sensitivity of naturally fractured reservoirs," in SPE Annual Technical Conference and Exhibition, Amsterdam, The Netherlands, 2014.

[12] Z. Chen, X. Liao, X. Zhao, S. Lyu, and L. Zhu, "A comprehensive productivity equation for multiple fractured vertical wells with non-linear effects under steady-state flow," Journal of Petroleum Science \& Engineering, vol. 149, pp. 9-24, 2017.

[13] A. K. M. B. Alam, M. Niioka, Y. Fujii, D. Fukuda, and J.i. Kodama, "Effects of confining pressure on the permeability of three rock types under compression," International Journal of Rock Mechanics and Mining Sciences, vol. 65, no. 1, pp. 4961, 2014.

[14] Y. Shen, G. Luan, H. Zhang, Q. Liu, J. Zhang, and H. Ge, "Novel method for calculating the effective stress coefficient in a tight sandstone reservoir," KSCE Journal of Civil Engineering, vol. 21, no. 6, pp. 2467-2475, 2017.

[15] W. Liu, J. Yao, Z. Chen, and W. Zhu, "An exact analytical solution of moving boundary problem of radial fluid flow in an infinite low-permeability reservoir with threshold pressure gradient," Journal of Petroleum Science and Engineering, vol. 175, pp. 9-21, 2019.

[16] X. Wang and J. J. Sheng, "Effect of low-velocity non-Darcy flow on well production performance in shale and tight oil reservoirs," Fuel, vol. 190, pp. 41-46, 2017.

[17] J. Wang, X. Wang, W. Xu, C. Lu, W. Dong, and C. Zhang, "Productivity analysis of volume fractured vertical well model in tight oil reservoirs," Mathematical Problems in Engineering, vol. 2017, no. 3, 14 pages, 2017.

[18] C. Lu, J. Wang, C. Zhang et al., "Transient pressure analysis of a volume fracturing well in fractured tight oil reservoirs," Journal of Geophysics and Engineering, vol. 14, no. 6, pp. 15091520, 2017.

[19] J. E. Warren and P. J. Root, "The behavior of naturally fractured reservoirs," Society of Petroleum Engineers Journal, vol. 3, no. 03, pp. 245-255, 1963. 
[20] L. Zhu, X. Liao, Z. Chen, and X. Cheng, "Pressure-transient analysis of a vertically fractured well in a tight oil reservoir with rectangular stimulated reservoir volume," SPE Production \& Operations, vol. 33, no. 04, pp. 697-717, 2018.

[21] Y. Su, G. Sheng, W. Wang, Q. I. Zhang, M. LU, and L. Ren, “A mixed-fractal flow model for stimulated fractured vertical wells in tight oil reservoirs," Fractals, vol. 24, no. 01, article 1650006, 2016(15 pages).

[22] Q. Zhang, S. Yuliang, W. Wang, and G. Sheng, "A new semianalytical model for simulating the effectively stimulated volume of fractured wells in tight reservoirs," Journal of Natural Gas Science and Engineering, vol. 27, no. part_P3, pp. 18341845, 2015.

[23] J. J. Guo, S. Zhang, L. H. Zhang, H. Qing, and Q. G. Liu, "Well testing analysis for horizontal well with consideration of threshold pressure gradient in tight gas reservoirs," Journal of Hydrodynamics, vol. 24, no. 4, pp. 561-568, 2012.

[24] H. Wang, Q. Ran, and X. Liao, "Pressure transient responses study on the hydraulic volume fracturing vertical well in stress-sensitive tight hydrocarbon reservoirs," International Journal of Hydrogen Energy, vol. 42, no. 29, pp. 1834318349, 2017.

[25] J. Ji, Y. Yao, S. Huang, X. Ma, S. Zhang, and F. Zhang, “Analytical model for production performance analysis of multifractured horizontal well in tight oil reservoirs," Journal of Petroleum Science and Engineering, vol. 158, pp. 380-397, 2017.

[26] Z. Wu, C. Cui, G. Lv, S. Bing, and G. Cao, "A multi-linear transient pressure model for multistage fractured horizontal well in tight oil reservoirs with considering threshold pressure gradient and stress sensitivity," Journal of Petroleum Science and Engineering, vol. 172, pp. 839-854, 2019.

[27] Z. Wu, C. Cui, Z. Wang, Y. Sui, P. Jia, and W. Tang, "Well testing model of multiple fractured horizontal well with consideration of stress-sensitivity and variable conductivity in tight gas reservoirs," Mathematical Problems in Engineering, vol. 2018, 13 pages, 2018.

[28] G. Sheng, Y. Su, W. Wang, F. Javadpour, and M. Tang, "Application of fractal geometry in evaluation of effective stimulated reservoir volume in shale gas reservoirs," Fractals, vol. 25, no. 04, article 1740007, 2017.

[29] T. Guo, S. Zhang, H. Ge, X. Wang, X. Lei, and B. Xiao, “A new method for evaluation of fracture network formation capacity of rock," Fuel, vol. 140, pp. 778-787, 2015.

[30] Q. Lei, W. Xiong, J. Yuang, Y. Cui, and Y.-S. Wu, “Analysis of stress sensitivity and its influence on oil production from tight reservoirs," in Eastern Regional Meeting, Lexington, KY, USA, 2007.

[31] Z. Chen, X. Liao, S. Lv et al., "Development of a trilinear flow model for carbon sequestration in depleted shale," in Paper SPE 176153 Presented at the SPE/IATMI Asia Pacific Oil \& Gas Conference and Exhibition, Nusa Dua, Bali, Indonesia, 2016.

[32] O. A. Pedrosa, "Pressure transient response in stress-sensitive formations," in The Paper Was Prepared for Presentation at the 56th California Regional Meeting of the Society of Petroleum Engineers Held in Oakland, Oakland, California, 1986. 

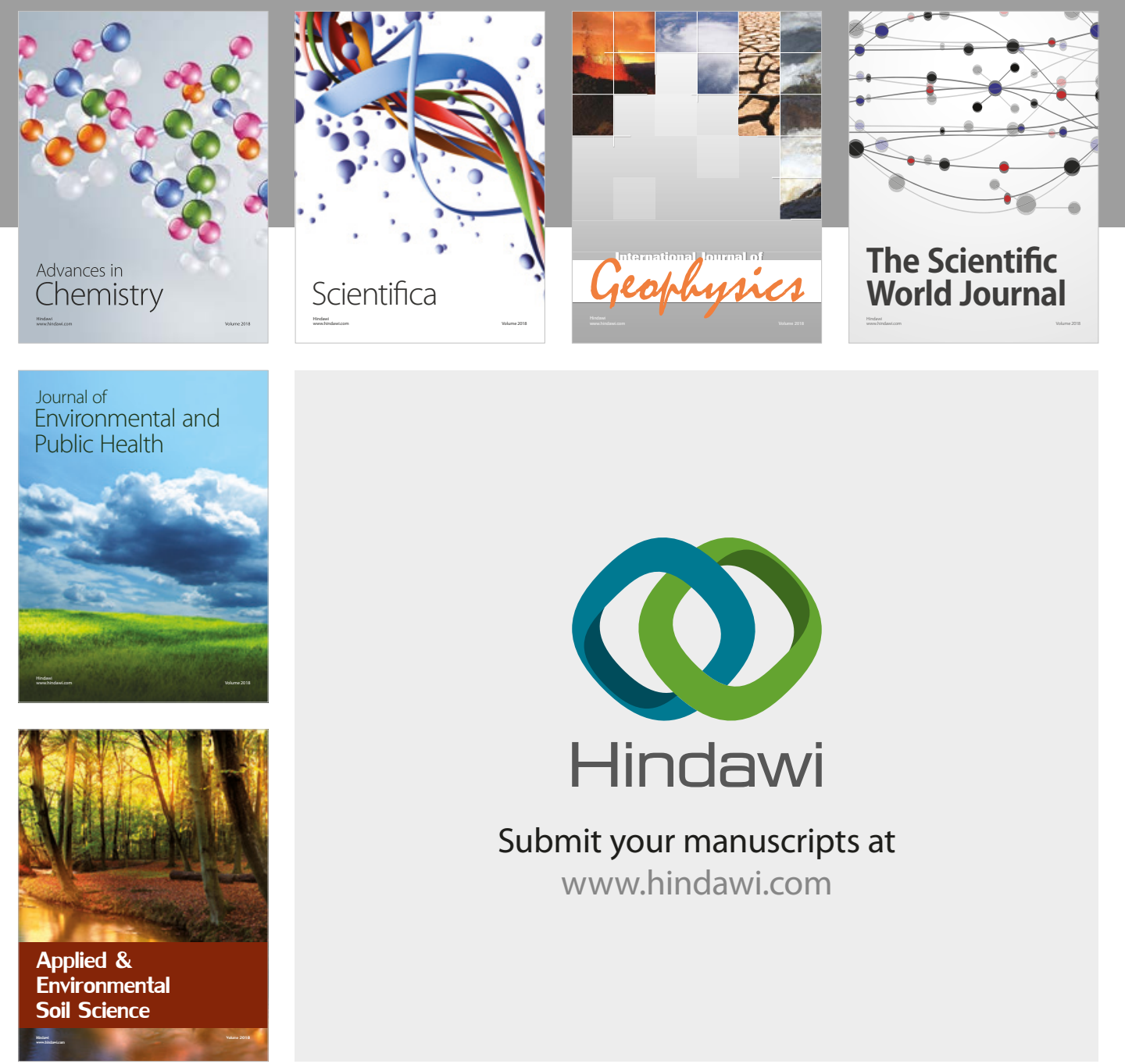

The Scientific

\section{World Journal}
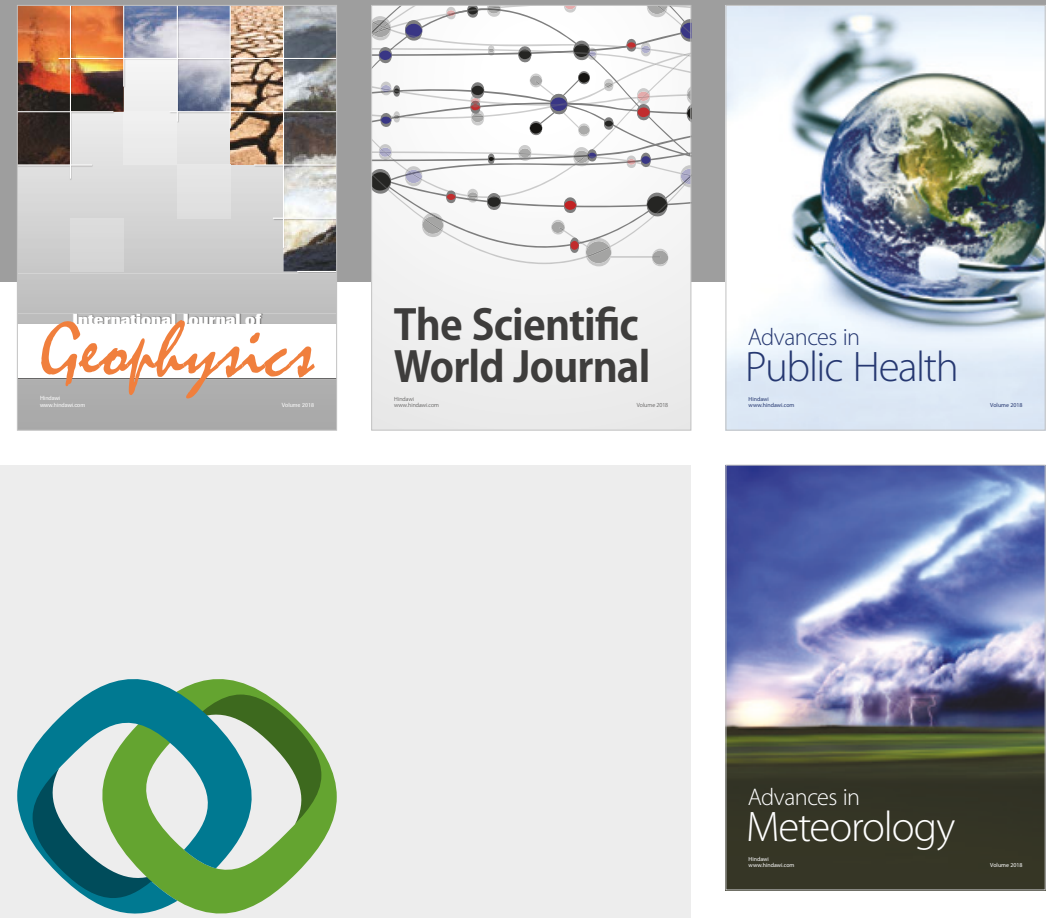

Advan

Public Health

\section{Hindawi}

Submit your manuscripts at

www.hindawi.com
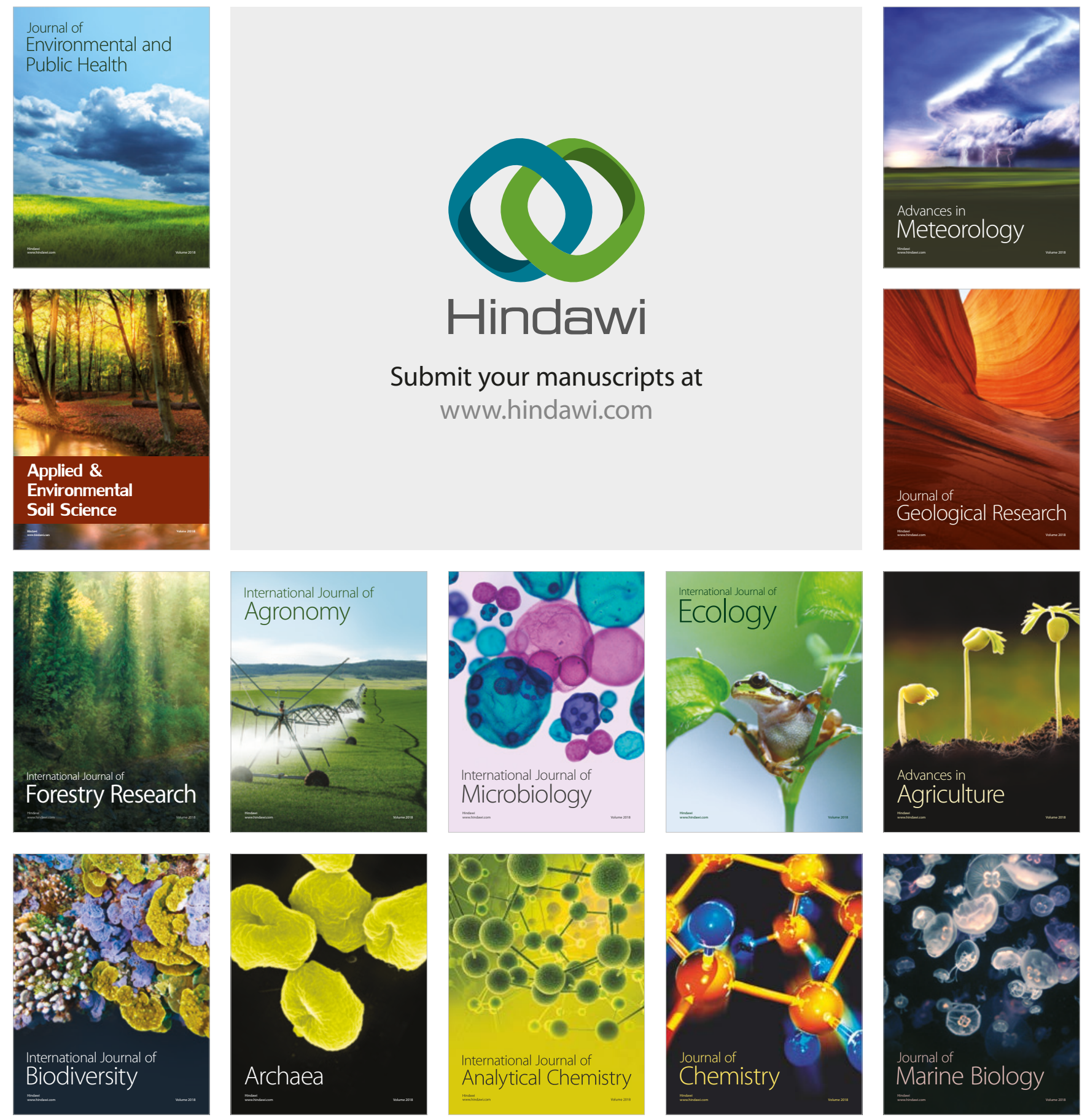